\title{
Les récits de métamorphoses du corps dans la nouvelle fantastique allemande du début du $\mathrm{xx}^{\mathrm{e}}$ siècle
}

Körperverwandlungen in der deutschsprachigen phantastischen Novellistik des Anfangs des 20. Jahrhunderts

Narratives of Bodily Metamorphosis in the Early Twentieth-Century German

Fantastic Short Story

Jean-Jacques Pollet

\section{CpenEdition}

Journals

Édition électronique

URL : https://journals.openedition.org/ceg/10071

DOI : 10.4000/ceg. 10071

ISSN : 2605-8359

\section{Éditeur}

Presses Universitaires de Provence

Édition imprimée

Date de publication : 20 janvier 2020

Pagination : 187-198

ISBN : 979-10-320-0253-7

ISSN : 0751-4239

\section{Référence électronique}

Jean-Jacques Pollet, «Les récits de métamorphoses du corps dans la nouvelle fantastique allemande du début du xxe siècle », Cahiers d'Études Germaniques [En ligne], 78 | 2020, mis en ligne le 28 octobre 2021, consulté le 01 février 2022. URL : http://journals.openedition.org/ceg/10071 ; DOI : https:// doi.org/10.4000/ceg.10071 


\title{
Les récits de métamorphoses du corps dans la nouvelle fantastique allemande du début du $\mathrm{XX}^{\mathrm{e}}$ siècle
}

\author{
Jean-Jacques POLLET \\ Université d'Artois, Textes et Cultures (EA 4028), Arras
}

Au sein du corpus constitué par les nouvelles fantastiques allemandes du début du $\mathrm{XX}^{\mathrm{e}}$ siècle (très largement portées par l'éditeur munichois Langen-Müller), nous isolons les récits de métamorphose, que nous entendons comme des récits qui racontent une transformation de tout ou partie du corps humain si considérable que l'être dont il s'agit n'est plus reconnaissable, au point d'appartenir, le cas échéant, à un autre règne (animalisation, végétalisation, minéralisation, réification).

Tous les récits de métamorphose, à quelque époque qu'ils appartiennent et quel que soit le genre littéraire dont ils relèvent, peuvent s'analyser selon la même grille ${ }^{1}$ : le substrat (la métamorphose affecte-t-elle le corps entier ou une partie du corps? est-elle subie, acceptée, volontaire?), l'agent (qui l'inflige? pour quels motifs?), le processus (quelle "technique" ? quelle cinétique - instantanée ou progressive?), le produit (quelle logique? amplification de certains traits de caractère du sujet ou logique inverse?). Chacun de ces quatre constituants renvoie lui-même à différentes variations, selon les genres littéraires et les époques.

La métamorphose est un thème privilégié du conte merveilleux et, aujourd'hui, de la science fiction. A-t-il une affinité particulière avec le fantastique? Si l'on considère que l'élément constitutif du récit fantastique est la mise en scène d'une "intranquillité" (Verunsicherung) rapportée au caractère inexplicable, selon les normes en vigueur du vraisemblable, de l'événement narré et/ou au caractère scandaleux de celui-ci selon les valeurs culturelles communément partagées, le thème de la métamorphose apparaît a priori comme particulièrement fécond, dans la mesure où il peut en principe jouer sur les deux registres ${ }^{2}$. Cette plasticité ouvre diverses nuances, depuis le fantastique-étrange (selon que le récit accentue prioritairement l'entorse aux règles de crédibilité que représente l'événement)

1. Voir Francis Berthelot, La Métamorphose généralisée, Paris, Nathan, 1993.

2. Il va de soi que ces normes de crédibilité autant que ces valeurs ne sont pas celles d'un lecteur "réel", mais celles qui sont incarnées à l'intérieur du texte lui-même par une instance (personnage, narrateur ou personnage-narrateur) censée arbitrer sur ce qui peut appartenir ou non à la réalité. 
jusqu'au fantastique-terrifiant ${ }^{3}$ (selon qu'il privilégie l'outrage que représente l'événement par rapport aux valeurs attachées à l'intégrité de la personne humaine). Les titres et sous-titres choisis par les écrivains qui nous concernent reflètent ces nuances en puisant dans un riche répertoire synonymique ${ }^{4}$.

Une échappée de la métamorphose hors du champ du fantastique proprement dit est illustrée par le récit de Franz Kafka qui porte ce titre 5 . La nouvelle respecte apparemment la loi du genre, à savoir, selon la célèbre formule de Roger Caillois, l'" irruption de l'inadmissible au sein de l'inaltérable légalité quotidienne ${ }^{6}$ ". Mais précisément, cette irruption brutale (" Un matin, au sortir d'un rêve agité, Grégoire Samsa s'éveilla transformé dans son lit en une véritable vermine $^{7}$ ") ne provoque ici ni étonnement ni effroi. Le scandale est regardé comme une chose naturelle et ne suscite aucune "intranquillité", ni de la part du sujet, qui est juste surpris et ne s'insurge pas véritablement, ni de la part de son entourage qui, n'ayant aucun moyen d'accéder à la conscience de Samsa, oublie progressivement qui il est pour ne plus voir en lui qu'une vermine, ratifiant la coupure schizophrénique instaurée (" il faut chercher à nous débarrasser de ça ${ }^{8}$ ). La métamorphose demeure inexplicable sous tous ses aspects : quel agent? quel motif? quelle technique? quelle logique? C'est qu'en vérité la métamorphose n'intéresse aucunement Kafka en elle-même, en tant que phénomène ; il ne retient que la substitution qui s'est opérée, ouvrant ainsi une lecture métaphorique qui dépasse le simple événement décrit.

\section{Manipulations de la chair}

$\mathrm{Au}$ sein de notre corpus se dégage un premier ensemble, qui réunit les récits où la métamorphose apparaît comme le fruit d'une chirurgie, d'une manipulation de la chair. Par rapport aux motifs d'“intranquillité", ceux-ci, en principe, n'offrent pas d'entorse aux règles de crédibilité intellectuelle ou, tout au moins, ne développent pas ce qui pourrait être un aspect "science-fiction" de la médecine ; ils passent sous silence la "technique" du processus. L"intranquillité" fantastique tient dès lors à l'agent de la métamorphose, à la motivation à laquelle celui-ci obéit, ainsi qu'au produit lui-même, en tant qu'atteinte à la valeur culturelle attachée à l'intégrité de la personne humaine.

3. Sur ce sujet du fantastique-terrifiant, voir Hans Richard Brittnacher, Ästhetik des Horrors. Gespenster, Vampire, Monster, Teufel und künstliche Menschen in der phantastischen Literatur, Frankfurt a. M., Suhrkamp, 1994. Brittnacher distingue cinq grands champs thématiques qui illustrent le fantastique-terrifiant : le fantôme, le vampire, le monstre, le diable, l'homme artificiel. La métamorphose est traitée dans le second chapitre ("Das Monstrum »).

4. Exemples : sonderbare Geschichten, seltsame Geschichten, bedenksame Historien, geheimnisvolle Geschichten, unheimliche Geschichten, das Grauen, die Besessenen...

5. Voir, sur ce sujet, Nicole Pelletier, "Le fantastique chez Kafka ", Austriaca 27,1998, p. 71-84.

6. Roger Caillois, Au cour du fantastique, Paris, Gallimard, 1965, p. 161.

7. Franz Kafka, La Métamorphose, trad. de l'allemand par Alexandre Vialatte, Paris, Gallimard (coll. " Folio ", 74), 1981 [1938], p. 5.

8. Ibid., p. 83. 
Ce type de récit est illustré par les premiers recueils de nouvelles de Gustav Meyrink, en particulier Orchidées. Histoires étranges (Orchideen. Sonderbare Geschichten, 1904) et Le Cabinet des figures de cire. Histoires étranges (Wachsfigurenkabinett. Sonderbare Geschichten, 1907).

Parmi ces textes, la nouvelle « Les Plantes du Dr. Cinderella » (" Die Pflanzen des Doktors Cinderella ") présente un caractère exemplaire. Voici un récit à la première personne dont le héros-narrateur nous raconte qu'à l'origine de " tous [1]es moments d'angoisse fantastique ${ }^{9}$ " qu'il a vécus ces dernières années, il y a une statuette égyptienne qu'il a ramenée jadis de Thèbes. Il s'exerce un soir à prendre la même attitude que celle de la statuette et a l'impression de " "sorti[r]" [...] de [son] corps ", de " [franchir] les limites ordinaires de la conscience" ". Plongé dans cette sorte d'état cataleptique, il se laisse entraîner à parcourir les ruelles sombres et tortueuses du vieux Prague; il s'arrête devant une vieille demeure, pousse la porte entrebâillée, descend l'escalier menant à la cave " comme [s'il] étai[t] ici dans [s]es murs ${ }^{11}$ " :

\begin{abstract}
Soudain, en tendant la main vers le mur, je touchai les échalas d'une sorte de treille comme on en emploie pour faire pousser les plantes grimpantes [...]. Le mur en face de moi était entièrement recouvert d'un lacis de veines rouge sang, duquel saillaient, comme des baies, des centaines d'yeux globuleux [...]. Au milieu de cet enchevêtrement chatoyaient d'innombrables et sinistres prunelles qui tour à tour dilataient leurs terrifiantes tubérosités de mûres sauvages pour me suivre du regard lorsque je passais devant elle [...]. Les plus grosses veines plongeaient leurs racines dans des fioles remplies de sang [...]. C'était comme une incroyable composition artistique à partir d'éléments pris sur des corps vivants, dépourvus de sensations humaines, réduits à une vie végétative. [...] Qui pouvait bien être le jardinier diabolique qui avait créé ce décor ${ }^{12}$ ?
\end{abstract}

Les manipulations du docteur Cinderella ne se confondent pas avec les expériences scientistes d'un docteur Frankenstein ou d'un docteur Moreau chez H. G. Wells. Il ne s'agit pas, pour lui, de concurrencer une démiurgie divine, mais de créer simplement ce qu'il appelle " un décor ". Son travail possède la gratuité de l'art pour l'art. Le médecin prométhéen a cédé la place à un " jardinier diabolique ", dont l'œuvre monstrueuse abolit la frontière entre le règne végétal et le règne animal, selon la même monstruosité que celle incarnée par l'orchidée, motif récurrent du fantastique début-de-siècle.

Ce docteur Cinderella a comme parent, dans une autre nouvelle intitulée " Thanatopraxie " (" Das Präparat »), un certain " démon persan " du nom de

9. Nous citons " Die Pflanzen des Doktors Cinderella " d'après la traduction que nous en avons proposée dans : La Fiancée du diable. Nouvelles fantastiques allemandes de 1900, réunies et présentées par Jean-Jacques Pollet, textes traduits par Jean-Jacques Pollet, Pierre Giraud, Guislain Riccardi, Paris, Albin Michel, 1994, p. 17-27; le sigle PC précède le numéro de page, ici PC 17. Les références au texte allemand renvoient à l'édition : Gustav Meyrik, " Die Pflanzen des Doktors Cinderella ", in Meyrink, Wachsfigurenkabinett. Sonderbare Geschichten, München, Albert Langen, 1908, abrégée en PDC. Ici PDC 187.

10. PC 20; PDC 191.

11. PC 22; PDC 194.

12. PC 23-25; PCD 196-198. 
Daraschekoh qui construit une effrayante horloge à partir d'organes humains - réification ornementale :

Au plafond de l'alcôve pendait, au bout d'une tige de cuivre, une tête aux cheveux blonds. La tige perçait le sommet du crâne. Le cou, enveloppé s'une écharpe de soie, surmontait les deux poumons rougeâtres, avec la trachée et les bronches. Le cœur, animé d'un mouvement rythmique, était entouré de fils dorés qui conduisaient à un petit appareil électrique disposé sur le sol. Les veines gonflées pompaient le sang dans deux vases à long $\mathrm{col}^{13}$.

Ce même Daraschekoh réapparaît dans le récit "Le Cabinet des figures de cire " ("Das Wachsfigurenkabinett "), où il présente dans son musée oriental " deux êtres créés à partir d'un même corps, sans en détruire la force vitale, simplement par adjonction d'organes animaux ${ }^{14}$ ". La métamorphose s'expose ici comme un spectacle, une attraction de fête foraine.

Ces récits pourraient n'être que banales ou douteuses histoires d'épouvante si la monstruosité était soigneusement tenue à distance, car imputée uniquement à l'exotisme appuyé de la figure démoniaque, agent de la métamorphose. Mais l'épilogue de l'histoire de Cinderella illustre comment la découverte du monstrueux ne reste pas extérieure au spectateur et simple objet de curiosité, mais induit au contraire chez celui-ci l' intranquillité" constitutive du fantastique. Lorsqu'il se réveille de sa torpeur, le héros-narrateur se retrouve au commissariat de police où il explique sa macabre découverte, donne l'adresse de la maison du crime. Il s'entend répliquer par le commissaire que " le docteur Cinderella est un grand savant, spécialiste d'égyptologie, connu pour ses expériences sur les plantes carnivores ${ }^{15}$. " Le greffier, à cet instant, vient lui rendre les cartes de visite tombées de sa poche. Elles portent le nom du docteur Cinderella. " Mais c'est vous-même! s'écrie le commissaire, vous devriez rester chez vous la nuit ${ }^{16}$ ! " Depuis cette aventure, le narrateur reste paralysé d'un côté du visage. Il a cherché en vain à retrouver la demeure maudite et " au commissariat, personne n'a souvenir de ce qui s'est passé ce soir-là ${ }^{17}$ ".

Autrement dit, la découverte de la métamorphose monstrueuse est bien une descente dans le propre inconscient du sujet, de même que l'ensemble de la géographie de Prague, avec ses ruelles labyrinthiques et ses façades baroques, dessine dans le roman meyrinkien un espace onirique, l'anthropomorphisation du paysage offrant en quelque sorte le pendant de la métamorphose des corps.

13. Gustav Meyrink, Des deutschen Spießers Wunderhorn, München, Langen-Müller, 1981 [1913], p. 186. C'est nous qui traduisons.

14. Gustav Meyrink, "Das Wachsfigurenkabinett ", in Meyrink, Wachsfigurenkabinett, p. 229. Traduction par nos soins.

15. PC 33 ; PDC 200-201.

16. PC, ibid. ; PDC 201.

17. PC 34; PDC 202. 


\section{Maléfices}

Un autre ensemble regroupe les récits où la métamorphose est le fruit d'un processus "magique", soit impersonnel (œuvre de la Nature, malédiction), soit incarné dans un personnage doué de pouvoirs supérieurs (sorciers, sorcières, magiciens, femmes fatales, etc.).

"L'Opale " ("Der Opal ${ }^{18}$ "), de Meyrink, réalise ce schéma. Ce sont les membres d'une expédition aux Indes qui, pour avoir forcé les portes d'un temple consacré à Shiva, sont retrouvés décapités, avec les yeux pétrifiés, métamorphosés en véritables opales. La métamorphose minérale est bien ici le châtiment pour un sacrilège culturel : l'Européen (l'Anglais, en l'espèce) est châtié pour son incrédulité et sa suffisance face à la religiosité orientale.

La métamorphose lycanthropique correspond également, traditionnellement, à un châtiment qui frappe celui qui a enfreint un tabou, pénétré un lieu interdit ${ }^{19}$. Hans Richard Brittnacher relève en particulier, au sein du corpus qui est le nôtre, deux modes singuliers du maléfice : une malédiction familiale qui poursuit le descendant d'une lignée de bourreaux (Hans Watzlik, "Die Wolfsleute ${ }^{20}$ "), une magie animiste qui frappe l'amant qui a volontairement revêtu la peau de bête pour posséder la femme aimée (Leonhard Stein, "Der Gorilla ${ }^{21}$ ").

Paul Busson, avec Les Buissons de Circée (Der Schuß im Hexenmoos ${ }^{22}$, 1923) livre un exemple relativement rare de métamorphose lycanthropique féminine qui s'écarte du schéma de l'intervention magique venant punir un sacrilège. Un garde-forestier prénommé Martin raconte son histoire liée à celle du château voisin, aujourd'hui tombé en ruine. Dès le premier jour où elle rentra de l'étranger, la veuve du comte, dont on disait qu'elle avait mené jadis une vie de débauche, qu'elle avait séduit de nombreux amants et provoqué leur mort, exerça sur lui une étrange fascination. Mais la comtesse rencontra bientôt, au cours de ses promenades solitaires, un braconnier fruste et sauvage qu'elle prit l'habitude de rejoindre dans son repaire. Martin, qui ne cessait de les traquer, fut congédié. Exactement à cette époque, toute la région fut mise en émoi par les massacres commis dans le bétail par un loup. Une battue fut organisée. Au cours de celle-ci, Martin repéra des traces, les suivit, constata qu'elles s'évanouissaient brusquement pour laisser place à des pas humains. Un soupçon l'envahit. Le lendemain, il suivit la comtesse dans les bois, la vit disparaître soudain dans un fourré. Il s'approcha, découvrit face à lui la bête féroce, saisit son fusil et tira :

18. Gustav Meyrink, "Der Opal ", in Meyrink, Des deutschen Spießers Wunderhorn.

19. Cf. Brittnacher, Ästhetik des Horrors, p. 119-121.

20. Récit paru dans le recueil : Hans Watzlik, Dämmervolk. Spukhafte Erzählungen, Leipzig, Staackmann, 1928.

21. Texte publié dans le volume : Toni Schwabe (éd.), Das Gespensterschiff. Ein Jahrbuch für die unheimliche Geschichte, Jena, Landhausverlag, 1920, p. 16-24.

22. Paul Busson, Der Schuß im Hexenmoos, Wien, Steyrermühl-Verlag, 1925. 
La comtesse gisait sur le sol, la main crispée sur son sein gauche d'où s'échappait un flot de sang - du sang humain, le sang d'une femme... "Loup-garou! " était-ce moi qui hurlais ce mot? Son corps se cabra en un sursaut sauvage, puis retomba ${ }^{23}$.

Busson associe le thème lycanthropique à l'image de la femme fatale : le portrait de la comtesse réunit quelques-uns des signes "démoniaques" classiques, que l'on rencontre également chez la femme-vampire : lèvres rouges, yeux émeraude, chevelure dorée.

La métamorphose animale - dont le processus est ici à vrai dire complètement éludé, et qui n'existe peut-être que dans l'imaginaire du narrateur - n'a pas valeur de châtiment, comme souvent dans la tradition populaire. Elle ne serait ici, pour autant qu'elle ait eu lieu, que la révélation d'une nature sauvage impérieuse, ultime excès de la passion charnelle qui lie la comtesse à son amant. Dégradation bestiale de la figure de la femme fatale.

Dans son épilogue, le récit introduit l'hypothèse de la folie du narrateur comme interprétation de la métamorphose qu'il relate. Après qu'il a tiré sur la comtesse, Martin perd connaissance. À son réveil, il raconte son aventure aux autorités, qui mettent ses propos extravagants sur le compte d'un esprit faible aveuglé par la jalousie, concluent au crime passionnel et le font interner. Il ne sortira que plusieurs années après, du jour où il comprendra qu'il peut recouvrer la liberté en renonçant à sa fable et en accréditant la thèse officielle. Et de conclure : "dans quelques heures, ce que je vous ai raconté vous paraîtra aussi invraisemblable qu'au médecin et à mes juges ${ }^{24}$ ". Cette explication rationnelle, si elle dissipe le mystère de la métamorphose en tant que phénomène, n'efface pas pour autant "l'intranquillité". Ne serait-elle qu'un fantasme, la métamorphose lycanthropique apparaîtrait encore, pour reprendre la définition classique, comme un scénario imaginaire figurant l'accomplissement d'un désir inconscient et ses interdits ${ }^{25}$.

Le récit sans conteste le plus original, dans cet ensemble de métamorphoses magiques, est celui de Hanns Heinz Ewers, "Journal d'un oranger " ("Aus dem Tagebuch eines Orangenbaumes ", 1905 ${ }^{26}$ ), qui présente une métamorphose végétale consentie et volontaire, vécue non pas comme une aliénation, mais comme un accomplissement.

Le héros-narrateur, interné depuis trois jours dans une clinique psychiatrique, rédige dans un carnet destiné au médecin-chef de l'institution quelques notes autobiographiques pour expliquer le motif qui l'a amené en ces lieux. Il souffrirait, prétend-on autour de lui, de "l'idée fixe d'être un oranger ${ }^{27}$ ". Il veut convaincre le médecin qu'il ne s'agit pas là d'un dérèglement de son imagination, mais d'une réalité, peut-être prodigieuse, mais incontestable.

23. Ibid., p. 3. C'est nous qui traduisons.

24. Ibid., p. 34.

25. Cf. Laplanche et Pontalis, Vocabulaire de la psychanalyse, Paris, Seuil, 1967.

26. Hanns Heinz Ewers, "Aus dem Tagebuch eines Orangenbaumes ", in Ewers, Das Grauen. Seltsame Geschichten, München, Georg Müller, 1908 (dans la suite T0 et numéro de page). Nous citons le texte français d'après la traduction de Pierre Giraud publiée dans La Fiancée du diable, p. 75-101; le numéro de page est précédé de l'abréviation JO.

27. J0 75; T0 187. C'est Ewers qui souligne. 
Tout commença le jour où il fit la connaissance, à l'occasion d'un bal de carnaval, d'une femme enchanteresse, une certaine Emy Steenhop. Cette " magicienne ${ }^{28}$ " menait grand train, tenait un salon fréquenté par tous les officiers de la garnison, tous à sa dévotion; le narrateur tomba sous son charme. Pour exaucer un désir de celle-ci, il prit l'habitude de lui apporter chaque soir des fleurs d'oranger cueillies dans ses serres; il lui dédiait des poèmes sur l'âme des fleurs et elle lui accorda de l'appeler Alcina - du nom de la fée qui punit ses soupirants en leur jetant un sort qui les fait prendre racine... Le langage des amants était un rêve végétal : il était l'arbre qui étendait sur elle l'ombre fraîche de ses bras, dont elle cueillait les fruits et respirait l'haleine parfumée... Il s'aperçut bientôt que son propre corps embaumait l'oranger, que ce parfum "éman [ait] de [s] a chair ${ }^{29}$ ". Puis vint l'ultime nuit où elle lui annonça son départ, ne lui laissant que ce billet : "Si tu m'aimes, tu dois parachever ta destinée. Oh, je reviendrai, je te reviendrai, mon bien-aimé. Je reposerai sous la fraîcheur de ton ombrage et te raconterai de douces légendes ${ }^{30}$. " Après une violente crise de désespoir qui a justifié, pour son entourage, son internement, il a aujourd'hui retrouvé la paix. Il sait qu'il ira jusqu'au bout de sa tâche. Il éprouve déjà de la peine à tenir sa plume, car ses doigts répugnent à se tenir serrés, ils s'écartent irrésistiblement tels des branches...

Bien qu'initiée par la femme fatale, la métamorphose est autant l'œuvre consciente et délibérée de sa "victime". Elle symbolise l'accomplissement suprême du précepte de l'artiste décadent, en vertu duquel la réalité se plie à la toute-puissance des pensées, la matière obéit à l'esprit, l'art est plus vrai que la vie. La métamorphose révèle une poétique, déjà inscrite dans les poèmes que le narrateur dédie à Alcina :

\footnotetext{
D'où vient que l'eucalyptus éveille en chaque artiste l'idée de bras féminins nus et tendus de nostalgie? Que l'asphodèle nous rappelle immanquablement la mort? Que la glycine nous suggère l'image d'une blonde fillette de pasteur, que l'orchidée nous rappelle le sabbat des sorcières et les messes noires? Précisément parce que de telles pensées vivent dans ces fleurs et ces arbres ${ }^{31}$.
}

Une variante, au sein de la thématique fantastique de la métamorphose "magique", est offerte par les histoires de réincarnation. À la condition que l'avatar ne corresponde pas simplement à un changement de personne, mais de catégorie dans le règne du vivant, on peut considérer qu'il s'agit d'une sorte de métamorphose post-mortem, le plus souvent adaptée librement du dogme de la métempsychose, en lien avec l'orientalisme dont nos écrivains se nourrissent abondamment à l'époque ${ }^{32}$. Il est néanmoins difficile de concilier cette transmigration avec "l'intranquillité" propre au fantastique, dans la mesure où la métempsychose,

28. J0 77; T0 188. C'est Ewers qui souligne.

29. JO 94 ; TO 192. C'est Ewers qui souligne.

30. JO 100; T0 187.

31. J0 90 ; TO 188. C'est Ewers qui souligne.

32. Voir, sur ce sujet, Jean-Jacques Pollet, Introduction à la nouvelle fantastique allemande, Paris, Nathan, 1997, p. 64-65. 
par définition, correspond à une promesse consolante de survivance de l'âme (d'autant que le dogme brahmanique fait des réincarnations successives un processus de purification). Le procédé le plus facile - et trop commode - pour introduire une dimension fantastique consiste dès lors, comme dans "L'Armoire mystérieuse " (" Das Geheimnis des Kleiderspinds ", 1908) de Kurt Münzer, à postuler chez le réincarné une sorte de mémoire d'outre-naissance qui le rend capable de se souvenir de ses vies antérieures et à lui prêter, dans la chaîne de ses avatars, une aventure terrifiante : la jeune femme de la nouvelle affirme péremptoirement que "si les hommes oublient les métamorphoses qu'ils ont traversées, il lui a été donné, à elle, de pouvoir suivre les transmigrations de son âme ${ }^{33}$ "; et de se souvenir que, dans une autre vie, elle s'est abandonnée à un amant vampirique qui, "gavé et enivré de son sang, s'effondra à ses pieds comme une bête repue ${ }^{34}$ ", avant qu'elle-même n'expirât et ne devînt hirondelle...

De la réincarnation végétale décrite dans " Le Mûrier de Hradschi Hussein " (" Hradschi Husseins Maulbeerbaum ", 19195), Busson ne retient qu'une leçon de vie positive : un sage ermite d'Orient passe ses jours à l'ombre d'un mûrier sous lequel il a jadis enterré son fils; les racines se sont nourries de son corps et c'est lui qui aujourd'hui vit, grandit et s'épanouit... La seule inquiétude vient de la présence d'Européens qui, indifférents à la culture locale, prétendent éradiquer le mûrier, en dépit des supplications du sage. Mais cette menace ne fait pas pour autant véritablement basculer le récit dans l'“intranquillité".

\section{Dévoiements ethnophobiques}

On ne peut passer sous silence les récits où la métamorphose, comme chez Karl Hans Strobl, est dévoyée pour véhiculer des ethnophobies aux relents nationalistes.

Sur le thème de la lycanthropie, "La Forêt d'Augustowo " (" Der Wald von Augustowo ${ }^{36}$ ") se déroule dans l'actualité de la guerre, sur le front de l'Est. Quelques soldats allemands égarés en pleine forêt trouvent refuge dans une chaumière habitée par une jeune femme et un colosse à l'allure presque bestiale. Ce dernier leur rapporte de sa chasse un animal encore sanguinolent dont ils font leur repas, en dépit de leur dégoût. Ils décident d'envoyer en reconnaissance l'un des leurs; mais celui-ci n'étant pas rentré à la tombée de la nuit, ils partent à sa recherche. Les seules traces qu'ils découvrent sont " comme des traces de loup "37 " qui les ramènent invariablement vers leur point de départ. C'est alors qu'une bête

33. Kurt Münzer, " Das Geheimnis des Kleiderspinds », in Münzer, Abenteuer der Seele, Berlin, G. Müller, 1908, p. 150. C'est nous qui traduisons.

34. Ibid., p. 291.

35. Paul Busson, "Hradschi Husseins Maulbeerbaum ", in Busson, Seltsame Geschichten, Graz/ Wien/ Leipzig, Kienreich (coll. " Bücherei österreichischer Schriftsteller ", 3), 1919.

36. Karl Hans Strobl, " Der Wald von Augustowo ", in Der Gespensterkrieg [anthologie], Stuttgart, Lese-Verlag, 1915.

37. Ibid., p. 112. 
monstrueuse se jette sur eux, avant d'être blessée à l'œil par un coup de pistolet et de disparaître dans l'obscurité. Lorsqu'ils rentrent plus tard à la chaumière, ils découvrent le colosse agonisant, le visage inondé de sang; un horrible soupçon les saisit...

Il y a ici une mise à plat, une version triviale du mythe de la lycanthropie, dépouillé de toute connotation théologique ${ }^{38}$. La référence au loup-garou n'est ici qu'une image pour signifier le caractère primitif, sauvage d'un certain type humain, le Slave :

Les babines retroussées découvraient une forte mâchoire, des dents éclatantes. De grosses mains noires et velues, comme des pattes de loup ${ }^{39}$.

Au-delà de cette zoologie, c'est dans la mise en scène elle-même de la monstruosité que tient le dévoiement raciste de la fiction. La monstruosité est ici pure altérité. Il y a d'un côté les braves soldats allemands égarés et de l'autre la Bête. Ceux-ci ne connaissent aucune "intranquillité ", ils ne doutent pas un instant d'eux-mêmes et de leur supériorité culturelle. Ils craignent simplement pour leur vie. Le fantastique cède ainsi à l'épouvante. Strobl met d'ailleurs ouvertement son propos raciste au service de la propagande belliciste, avec cette réflexion prêtée à l'un des soldats allemands, en arrivant dans la chaumière isolée :

Nous voilà manifestement au cœur de la culture russe... Plût à Dieu que Monsieur Poincaré et ces messieurs de l'Académie française pussent contempler avec nous, dans toute leur splendeur, leurs frères d'armes... ${ }^{40}$

"La Faute du mousquetaire Thomas Feuerweiss " (" Die Verantwortung des Musketiers Thomas Feuerweiss ${ }^{41} "$ ) met à distance le terrifiant par le double écran du recul historique et de l'exotisme : l'histoire se passe au XVII siècle, dans une colonie africaine. Un soldat originaire du Brandebourg se prend de passion pour une esclave maure, Ajebba, "belle sorcière ${ }^{42}$ " qui nourrit une haine féroce à l'égard de son maître étranger. En dépit de la surveillance de celui-ci, la jeune esclave s'échappe chaque nuit, tandis que son maître se trouve frappé d'une étrange paralysie. Les châtiments qu'il lui inflige attisent encore la haine d'Ajebba. Un soir, il se voit capturé par des indigènes et au cours d'un rituel magique mené par Ajebba en personne, il se trouve plongé dans un état léthargique, " comme s'il se transformait en un vulgaire bout de bois ${ }^{43}$ "; il reste ainsi prisonnier six années durant, statuette morte-vivante exposée aux yeux des indigènes, idole d'un culte sauvage dont la grande prêtresse, Ajebba,

38. Voir Klaus Völker (éd.), Von Werwölfen und anderen Tiermenschen, München, Hanser, 1972.

39. Strobl, " Der Wald von Augustowo ", p. 108. Traduction par nos soins.

40. Ibid., p. 95.

41. Karl Hans Strobl, " Die Verantwortung des Musketiers Thomas Feuerweiss ", in Strobl, Beelzebubs Meerschaumkopf. Phantastische Novellen, Wien/ Berlin/ Leipzig/ Bern, Europäischer Verlag, 1924.

42. Ibid., p. 18.

43. Ibid., p. 22. 
ordonne les débordements cruels et orgiaques. Il ne sera libéré que par l'arrivée des soldats européens.

La métamorphose apparaît bien comme le châtiment de l'Allemand pour sa coupable passion pour la jeune fille indigène, qui incarne le déchaînement sexuel, la licence, la cruauté. L'immobilisation liée à la métamorphose végétale reçoit d'ailleurs un sens ambigu : en même temps qu'elle punit l'Européen, elle le protège d'une certaine manière du déchaînement orgiaque, dont il reste simple spectateur; la torture est disproportionnée par rapport à la faute, à moins qu'il soit impardonnable, pour un bon Allemand, d'aimer une esclave maure... Son récit constitue le plaidoyer qu'il prononce devant le tribunal militaire, devant lequel il doit répondre de l'accusation de désertion et qu'il conclut par ses mots : " Je fais confiance à mes juges ${ }^{44} \ldots$ " Justice lui sera rendue. L'idéologie colonialiste est intacte.

\section{Subversions grotesques}

Il reste enfin à distinguer une dernière catégorie de récits, qui utilisent le thème de la métamorphose comme instrument d'une subversion grotesque.

Sans nous engager dans le débat théorique sur la proximité entre le fantastique et le grotesque, retenons que celle-ci est abondamment théorisée par nos écrivains, notamment à travers diverses préfaces d'anthologies de nouvelles, sous le signe du double héritage revendiqué d'E.T.A. Hoffmann et Edgar Allan Poe ${ }^{45}$. On retiendra qu'à la différence du récit fantastique stricto sensu qui se doit de respecter, au moins provisoirement, le vraisemblable avant d'y ouvrir une brèche, l'histoire grotesque se situe d'emblée au-delà de ce code, selon ce que Mikhaïl Bakhtine appelle "l'exceptionnelle liberté d'invention ${ }^{46}$ ", afin de mettre en scène une incongruité (qui est à la fois proche et différente de l'“intranquillité") en charge d'une portée subversive (Bakhtine nomme anakrisis cette manière de mettre à l'épreuve une vérité à travers l'incongruité). Le thème de la métamorphose peut évidemment se prêter à cette stratégie.

"Le Soldat brûlant " (" Der heiße Soldat "), le premier récit publié par Meyrink dans la revue munichoise Simplicissimus en 1901, illustre le procédé. L'histoire se déroule en Orient, lors d'une campagne militaire. Blessé au combat, un soldat, après avoir absorbé un mystérieux breuvage préparé par un fakir, est transporté dans un hôpital militaire. Sa fièvre atteint bientôt un tel degré que l'on doit le

44. Ibid., p. 26.

45. Voir par exemple Das lustige Gespensterbuch, München, G. Müller, 1915 (préface de G. Meyrink) ; Das unheimliche Buch, München, G. Müller, 1914 (préface de K. H. Strobl) ; Das Gespensterbuch, München, G. Müller, 1915 (préface de G. Meyrink) ; Das Buch der Grotesken, München, G. Müller, 1914 (préface de F. Lorenz). Citons également l'essai de Jakob Elias Poritzky, " Die groteske Dichtung ", Berliner Tageblatt, 13 avril 1916.

46. Mikhaïl Bakhtine, L'Euvre de François Rabelais et la culture populaire au Moyen-Âge et sous la Renaissance, trad. du russe par Andrée Robel, Paris, Gallimard (coll. " Tel ", 70), 1970 [1965], p. $55 \mathrm{sq}$. 
saisir avec des pinces, que les objets qui l'entourent s'enflamment spontanément, que l'eau dont on l'asperge se transforme en vapeur, avant qu'un père jésuite ne jette sur son corps un vêtement d'amiante, tandis que le médecin militaire qui assiste à la scène, pressé d'émettre une opinion, se contente d'expliquer le phénomène " par un dérèglement du centre thermique cervical, soumis aux lois de l'hérédité ${ }^{47}$."

L'anakrisis permet de discréditer les témoins de la scène, en l'occurrence militaires bornés et médecins positivistes, cibles favorites de la satire meyrinkienne de la société wilhelminienne.

Dans le même esprit, la métamorphose grotesque de " La Mort violette " ("Der violette Tod", 1902) fournit à Meyrink le prétexte d'une forme de parabole. Un explorateur anglais, Sir Hannibal Roger Thornton, veut aller étudier les mœurs d'une tribu méconnue et redoutée : on raconte que ces indigènes idolâtrent, sous forme de paon, un être satanique qui leur aurait révélé, au cours des siècles, les secrets de la magie noire, et " en particulier appris à articuler certains sons qui leur permettent d'anéantir sur le champ un ennemi, aussi fort soit-il ${ }^{48}$ ". Après vingt-cinq jours de marche, il arrive enfın dans l'inaccessible contrée. Face à lui s'avance une centaine d'hommes, conduits par leur chef. Sur un geste de celui-ci, les Tibétains se bouchent les oreilles et poussent un cri puissant :

La silhouette de Sir Roger perdit son maintien, sa tête s'allongea en pointe, toute la masse de son corps, comme liquéfiée, se recroquevilla et à l'endroit où, quelques instants auparavant, se tenait l'intrépide Anglais, il n'y avait plus maintenant qu'une espèce de quille d'un violet clair, de la forme d'un pain de sucre ${ }^{49}$.

Il s'agit du thème classique de la métamorphose interprétée comme châtiment pour une curiosité déplacée, qui porte ici une satire du stéréotype de l'Anglais, globe-trotter colonisateur armé d'un scepticisme dédaigneux devant les superstitions indigènes. Mais l'épilogue de la nouvelle élargit la perspective au-delà de cette simple satire. Étant sourd, le valet de Sir Thornton a échappé à la métamorphose grotesque. Il a toutefois lu sur les lèvres des Tibétains la formule magique, l'a transcrite dans un rapport que la presse internationale diffuse. "La mort violette " se répand ainsi dans le monde, n'épargnant que les sourds et les muets. La moitié de l'humanité disparaît en une semaine : "Quelques années plus tard, vers l'année 1950, une nouvelle race d'hommes, tous sourds-muets, peuple le globe. Us et coutumes, rangs et fortunes sont bouleversés. Un médecin spécialiste des maladies otiques dirige le monde ${ }^{50} \ldots$. . Meyrink a la conviction que la culture occidentale, aveuglée par ses certitudes positivistes, est promise à la ruine. Il verra dans l'éclatement du conflit mondial la réalisation de cette vision prophétique.

47. C'est nous qui traduisons.

48. Gustav Meyrink, " Der violette Tod ", in Meyrik, Des deutschen Spießers Wunderhorn, p. 334. Traduction par nos soins.

49. Ibid., p. 335.

50. Ibid., p. 339. 
Dans la toute dernière partie de son œuvre, à travers quelques textes épars publiés vers 1930, Meyrink fait un retour plein d'autodérision sur ses débuts littéraires et, dans ce cadre, revient avec humour sur le thème de la métamorphose, avec " Le Cacatoès blanc du Dr. Haselmayer " " Dr. Haselmayers weißer Kakadu ${ }^{51}$ »). Le docteur Sacroboso Haselmayer, vieil original qui vivait à l'écart du monde, au milieu de ses livres anciens, dans un palais baroque de Prague, est mort, dit-on, le matin de son quatre-vingtième anniversaire. Mais le narrateur a une autre théorie. Il veut croire que le docteur Haselmayer n'est pas vraiment mort, mais qu'il s'est métamorphosé en un oiseau, un cacatoès blanc. D’abord parce que la police n'a pas retrouvé son corps, lors de la perquisition, bien que toutes les portes fussent fermées de l'intérieur. Ensuite parce que son visage, en vieillissant, ressemblait de plus en plus à la tête de ce volatile et que, depuis longtemps, il ne sortait plus de chez lui que pour acheter des graines de tournesol, si bien que les voisins pensaient " qu'il avait chez lui un oiseau ${ }^{52}$ " (en allemand, einen Vogel haben signifie, au sens métaphorique, "être un peu fou "). Enfın parce que le narrateur a retrouvé un cahier de la main du docteur " dont la couverture portait d'évidentes traces de coups de bec ", où il avait consigné cette réflexion :

Dans l'ancienne Égypte déjà, on savait que l'âme habite le corps comme un oiseau dans sa cage. Aujourd'hui, les gens estiment que c'est là un langage symbolique. C'est une ineptie : il faut prendre la formule à la lettre ${ }^{53}$.

Le narrateur est celui qui, contre le sens commun, choisit de prendre la formule métaphorique à la lettre - il incarne en quelque sorte une autre folie, une folie "littéraire", qu'il assume : " il y a une autre interprétation, celle à laquelle je crois. Je veux la dire, au risque de passer pour fou ${ }^{54}$ ".

Dans la mesure où elle consiste en une substitution du produit au sujet, la métamorphose est en soi un phénomène métaphorique, et donc éminemment littéraire, que revendique Meyrink en tant que tel.

Ainsi la métamorphose apparaît bien, au terme de nos lectures, comme un thème de prédilection de la nouvelle fantastique allemande du début $d u$ $\mathrm{XX}^{\mathrm{e}}$ siècle, qu'elle investit sous de multiples aspects (fantastique-étrange, fantastique-terrifiant, fantastique-grotesque) pour promouvoir, en réaction aux mièvreries de la Heimatliteratur (littérature du terroir) et aux épigones du naturalisme, une littérature qu'elle veut " à rebours ", à la fois esthétisante, spiritualiste et subversive, mais qui l'expose, chez certains, au risque de trivialisation et de dévoiements idéologiques.

51. Gustav Meyrink, « Dr. Haselmayers weißer Kakadu », Dantziger Neueste Nachrichten, 30 janvier 1930, repris dans Das Haus zur letzten Latern, München, Langen-Müller, 1973.

52. Meyrink, " Dr. Haselmayers weißer Kakadu », in Das Haus zur letzten Latern, p. 177. Traduction par nos soins.

53. Ibid., p. 178.

54. Ibid., p. 177. 